\title{
Autoimmune Diseases Associated with Primary Biliary Cholangitis
}

\author{
Sanae Lajouad*, Imane Benelbarhdadi, Camelia Berhili, Nawal Lagdali, Mohamed Borahma, Fatima Zahra Ajana
}

Department of Digestive Diseases, Medicine C, University Hospital Center IBN SINA University Mohamed V, Rabat-Morocco

\begin{tabular}{ll}
\hline DOI:10.36348/SJMPS.2019.v05i09.004 & | Received: 29.08.2019| Accepted: 05.09.2019| Published: 11.09 .2019 \\
*Corresponding author: Sanae Lajouad &
\end{tabular}

\section{Abstract}

Primary biliary cholangitis (PBC) is a chronic cholestatic liver disease; the autoimmune mechanism seems the most likely. As a result, $\mathrm{PBC}$ is frequently associated with other autoimmune diseases. The goal of our work is to determine the prevalence and type of autoimmune diseases associated with PBC and to assess their impact on the prognosis of the disease. Materials and methods: This is a retrospective study over a period of 22 years (1998-2019) including all patients followed for CBP. In all these patients, we systematically looked for: autoimmune hepatitis, dysthyroidism, and type 1 diabetes, dry syndrome, and celiac disease, dermatological and joint damage. The statistical analysis of the data was done using the SPSS software. The comparison of the biochemical response to ursodeoxycholic acid between patients with isolated CBP and those with CBP associated with autoimmune pathology was performed using the Chi2 test. A combination of variables was considered statistically significant if $\mathrm{p}<0.05$. Results: 90 patients ( 85 women and 5 men) followed for CBP were collected. The average age was 49+/-12.3 years. Of these patients, 36 patients $(42.9 \%)$ had an autoimmune disease associated with PBC. The discovery of these autoimmune diseases preceded the diagnosis of PBC in 9 cases $(27.3 \%)$ and was concomitant in the remaining cases. Autoimmune hepatitis was found in 10 patients (12\%), defining an overlapping syndrome. Other diseases were Hashimoto's thyroiditis $(\mathrm{n}=9)$, basedow $(\mathrm{n}=1)$ dry syndrome $(\mathrm{n}$ $=10)$, celiac disease $(\mathrm{n}=3)$, insulin-dependent diabetes $(\mathrm{n}=2)$, systemic scleroderma $(\mathrm{n}=1)$, rheumatoid arthritis $(\mathrm{n}=$ 1), Addisson disease $(n=1)$ Psoriasis $(n=1)$ vitiligo $(n=1)$. The comparison of the biochemical response to ursodeoxycholic acid between patients with isolated CBP and those with CBP associated with autoimmune pathology was statistically non-significant with $\mathrm{p}=0.67$. Conclusion: In our series, the prevalence of autoimmune diseases associated with PBC was $40 \%$. These diseases were dominated by autoimmune hepatitis, Hashimoto's thyroiditis and dry syndrome. Although their association does not appear to alter the prognosis for CBP or the response to AUDC, their screening must be systematic in order to initiate early and appropriate treatment. Although ursodeoxycholic acid is beneficial in the treatment of primary biliary cirrhosis, it has had no measurable effect on the autoimmune disorders associated with the disease.

Keywords: Primary biliary cholangitis; autoimmune diseases; Paris 2 criteria; ursodeoxycholic acid.

Copyright @ 2019: This is an open-access article distributed under the terms of the Creative Commons Attribution license which permits unrestricted use, distribution, and reproduction in any medium for non-commercial use (NonCommercial, or CC-BY-NC) provided the original author and source are credited.

\section{INTRODUCTION}

Primary biliary cholangitis $(\mathrm{PBC})$ is a chronic cholestatic liver disease; the autoimmune mechanism seems the most likely. As a result, PBC is frequently associated with other autoimmune diseases. The etiology of PBC is unknown; however, it is assumed to be related to autoimmune mechanisms, supported by the discovery of lymphocytes in portal inflammatory infiltrates, the detection of characteristic antimitochondrial antibodies in about $90 \%$ of patients and the presence of associated autoimmune pathologies $[1,2]$. The most common associated autoimmune disorders include autoimmune hepatitis, Hashimoto's thyroiditis and dry syndrome. Controlled clinical trials have shown that ursodeoxycholic acid (UDCA) improves the biochemical and clinical characteristics of
PBC [3, 4]. However, there have been no reports of the effects of ADUC on autoimmune disorders associated with PBC. The goal of our work is to determine the prevalence and type of autoimmune diseases associated with $\mathrm{PBC}$ and to assess their impact on disease prognosis and therapeutic response to AUDC; therefore, we reviewed the data from our previously reported trial to determine whether disease improvement was also associated with improvement in these associated autoimmune diseases [4].

\section{MATERIAL AND METHOD}

It is a retrospective study over a period of 22 years (1998-2019) including all patients followed for PBC. In all our patients, we systematically searched for: autoimmune hepatitis (according to the Paris criteria the 
presence of 2 of the 3 criteria: ALAT $5 *$ LSN; serum IgG level $2 *$ LSN or presence of AML; hepatic biopsy showing moderate to severe interface hepatitis) dysthyroidism (thyroid check-up, antithyroid autoantibodies), type 1 diabetes, dry syndrome (ophthalmological examination, Schirmer test and lip biopsy if clinical signs are present), celiac disease (duodenal biopsies) and dermatological or joint involvement (clinical examination). The statistical analysis of the data was done using the SPSS software. The comparison of the biochemical response to ursodeoxycholic acid between patients with isolated CBP and those with CBP associated with autoimmune pathology was performed using the Chi2 test. a combination of variables was considered statistically significant if $\mathrm{p}<0.05$.

\section{RESULTS}

90 patients (85 women and 5 men) followed for CBP were collected. The average age was $49+/-12.3$ years. Of these patients, 36 patients $(42.9 \%)$ had an autoimmune disease associated with PBC. The discovery of these autoimmune diseases preceded the diagnosis of PBC in 9 cases $(27.3 \%)$ and was concomitant in the remaining cases. Autoimmune hepatitis was found in 9 patients $(10 \%)$, defining an overlapping syndrome. Other diseases were Hashimoto thyroiditis in $10 \%(n=9)$, basedow $(n=1)$ dry syndrome in $11.1 \%(\mathrm{n}=10)$, celiac disease in $3.3 \%(\mathrm{n}=3)$, insulin-dependent diabetes $(\mathrm{n}=1)$, systemic scleroderma $(\mathrm{n}=1)$, rheumatoid arthritis $(\mathrm{n}=1)$, Addisson disease $(n=1)$ Psoriasis $(n=1)$ vitiligo $(n=1)$ (Figure 1). Patients with and without these associated conditions were clinically similar, age, gender and response to AUDC as shown in Table 1. The comparison of the biochemical response to ursodeoxycholic acid between patients with isolated CBP and those with CBP associated with autoimmune pathology was statistically non-significant with $\mathrm{p}=0.67$ Table 2 .

Table-1: Comparison of Other Clinical Features in Primary Biliary Cirrhosis Patients with and Without Associated Autoimmune Conditions

\begin{tabular}{|c|c|c|c|}
\hline & $\begin{array}{l}\text { Whihout Associated } \\
\text { Conditions }\end{array}$ & $\begin{array}{l}\text { With Associated } \\
\text { Conditions }\end{array}$ & $p$ Value \\
\hline Age (yx) & $48,5+/-9,2$ & $46,4+/-8,3$ & 0.12 \\
\hline Gender & & & 0.10 \\
\hline Male & 2 & 2 & \\
\hline Eemale & 46 & 34 & \\
\hline icterus. & 30 & 21 & 0.82 \\
\hline asthenia & 8 & 11 & 0.13 \\
\hline pruritus. & 27 & 16 & 0.34 \\
\hline Bilizubun elexated & 15 & 12 & 0.68 \\
\hline sytolysis & 26 & 17 & 0.82 \\
\hline $\begin{array}{l}\text { Paris II (good } \\
\text { response) }\end{array}$ & 20 & 14 & 0.67 \\
\hline
\end{tabular}

Table-2: Comparison of the biochemical response to ursodeoxycholic acid between in Primary Biliary Cirrhosis Patients With and Without Associated Autoimmune Conditions:

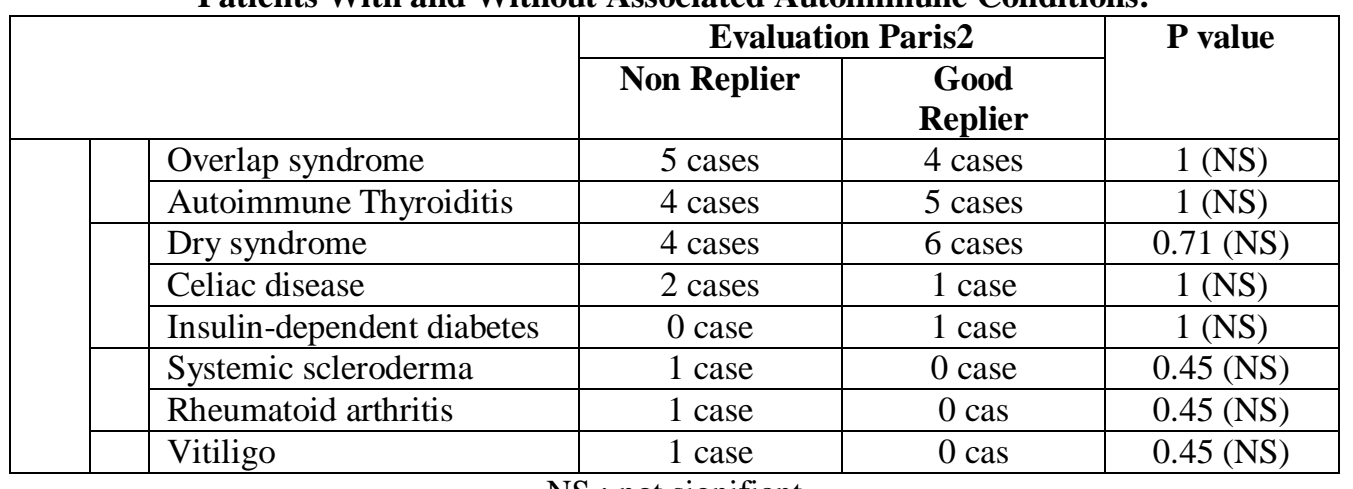

NS : not signifiant

\section{DISCUSSION}

Primary biliary cirrhosis (PBC) is a chronic cholestatic cholangitis of unknown etiology. It is morphologically characterized by portal inflammation and necrosis of small and medium size bile duct cells. This disease can frequently coexist with other 
autoimmune diseases; the treatment is based on ursodeoxycholic acid (AUDC) [5].

Controlled clinical trials have shown that UDCA improves the biochemical and clinical characteristics of PBC [3, 4] and significantly improves survival without liver transplantation [6]. There is no information on the effects of ADUC on associated autoimmune diseases such as dry syndrome, psoriasis, vitiligo, Basedow, Addison's disease, scleroderma, insulin-dependent diabetes, rheumatoid arthritis, celiac disease or Hashimoto's thyroiditis.

In our experience, AUDC has not influenced the resolution or prevented the development of these autoimmune diseases, despite the improvement made during PBC, as previously reported [4]. Previous publications have described the prevalence of associated conditions at the time of diagnosis of PBC, including the four most common conditions assessed in this study (dry syndrome, overlap syndrome, celiac disease and autoimmune thyroiditis), with values comparable to those of our study population.

The prevalence of autoimmune diseases is similar to that of other reports. Culp et al (1) described 113 patients, $84 \%$ of whom had at least one associated autoimmune disease, compared to $40 \%$ in our study. In other studies comparable to ours by Zukowski et al. [7] out of 180 patients, the prevalence of autoimmune diseases associated with PBC was $43 \%$ and in the study by Gharbi et al. the prevalence was $41 \%$. We compared the study of Zukowski et al. [7], Gharbi et al. [5] and our study found that Sec syndrome was the most frequent, occurring in $58 / 180$ patients $(32 \%)$ in the study of Zukowski et al. against $12.1 \%$ in the study of Gharbi et al. [5] versus $11.1 \%$ in our study, rheumatoid arthritis in $9 \%$ of patients versus $1.1 \%$ in our study and in the Gharbi et al. study [5] and hypothyroidism in $12 \%$ in both studies $[7,5]$ versus $10 \%$ in our series.

Previous studies in a smaller number of patients have suggested that the presence of associated autoimmune diseases predicts a poorer prognosis than that observed in patients without these conditions [8,9]. We found no association between the severity of PBC or its evolution and the presence and absence of these specific autoimmune characteristics. We conclude that UDCA does not influence the progression of dry syndrome, celiac disease, polyarthritis or Hashimoto's thyroiditis in patients with PBC as reported in the study by Zukowski et al. [7]. Physicians caring for these patients should be aware that treatment with UDCA does not influence the clinical course of these associated diseases and should therefore provide symptomatic care for these other associated autoimmune disorders.

\section{CONCLUSION}

In our series, the prevalence of autoimmune diseases associated with PBC was $40 \%$. These diseases were dominated by autoimmune hepatitis, Hashimoto's thyroiditis and dry syndrome. Although their association does not appear to alter the prognosis for CBP or the response to AUDC, their screening must be systematic in order to initiate early and appropriate treatment. Although ursodeoxycholic acid is beneficial in the treatment of primary biliary cirrhosis, it has had no measurable effect on the autoimmune disorders associated with the disease.

\section{REFERENCES}

1. Culp, K. S., Fleming, C. R., Duffy, J., Baldus, W. P., \& Dickson, E. R. (1982, June). Autoimmune associations in primary biliary cirrhosis. In Mayo Clinic Proceedings (Vol. 57, No. 6, pp. 365-370).

2. Gershwin, M. E., \& Mackay, I. R. (1995). New knowledge in primary biliary cirrhosis. Hospital Practice, 30(8), 29-36.

3. Jorgensen, R. A., Dickson, E. R., Hofmann, A. F., Rossi, S. S., \& Lindor, K. D. (1995). Characterisation of patients with a complete biochemical response to ursodeoxycholic acid. Gut, 36(6), 935-938.

4. Lindor, K. D., Dickson, E. R., Baldus, W. P., Jorgensen, R. A., Ludwig, J., Murtaugh, P. A., ... \& Lesage, G. (1994). Ursodeoxycholic acid in the treatment of primary biliary cirrhosis. Gastroenterology, 106(5), 1284-1290.

5. Lindgren, S., Manthorpe, R., \& Eriksson, S. (1994). Autoimmune liver disease in patients with primary Sjögren's syndrome. Journal of hepatology, 20(3), 354-358.

6. Heathcote, E. J., Lindor, K. D., Poupon, R., CauchDudek, K., Dickson, E. R., Trout, R., \& Poupon, R. E. (1995). Combined analysis of French, American and Canadian randomized controlled trials of ursodeoxycholic acid therapy in primary biliary cirrhosis. Gastroenterology, 108(4), A1082.

7. Harada, N., Dohmen, K., Itoh, H., Ohshima, T., Yamamoto, H., Nagano, M., ... \& Ishibashi, H. (1992). Sibling cases of primary biliary cirrhosis associated with polymyositis, vasculitis and Hashimoto's thyroiditis. Internal medicine, 31(2), 289-293.

8. Beswick, D. R., Klatskin, G., \& Boyer, J. L. (1985). Asymptomatic primary biliary cirrhosis: a progress report on long-term follow-up and natural history. Gastroenterology, 89(2), 267-271.

9. Nyberg, A., \& Lööf, L. (1989). Primary biliary cirrhosis: clinical features and outcome, with special reference to asymptomatic disease. Scandinavian journal of gastroenterology, 24(1), 57-64. 